\title{
POSITRON ANNIHILATION IN CHLOROPOLYSTYRENES
}

\author{
A. Baranowski ${ }^{a}$, M. Dębowska ${ }^{a}$, K. Jerie ${ }^{a}$, G. Mirkiewicz ${ }^{b}$, \\ J. RUDZIŃSKA-GIRULSKA ${ }^{a}$ AND R.T. SIKORSKI ${ }^{b}$ \\ anstitute of Experimental Physics, Wrochaw University \\ pl. Maxa Borna 9, 50-204 Wroclaw, Poland \\ ${ }^{b}$ Institute of Organic and Polymer Technology, Technical University of Wroclaw \\ Wybrzeże Wyspianskiego 27, 50-370 Wrocław, Poland
}

Results of angular correlation of annihilation radiation and positron annihilation lifetime measurements are presented for atactic polystyrene and chloropolystyrenes. The inhibition of positronium formation follows the chlorine introduction as the decrease in intensities of the narrow component in angular correlation of annihilation radiation curves anci of the longest-lived component in positron annihilation lifetime spectra prove. In general, the chlorine "activity" towards positrons seems to depend on its position in the polymer structure. The annihilation rate distribution as well as the radius and volume distributions of free-volume sites where positronium is assumed to be formed obtained with the use of CONTIN program for the raw sample in the 2nd series are given additionally.

PACS numbers: 71.60.tz, 78.70.Bj, 81.20.Sh, 82.30.Hk

\section{Introduction}

The recent increase in number of papers on studies of polymers with the use of positron annihilaton spectroscopy (PAS) reflects the fact that the latter is the most novel technique and very useful for the study of polymers at a molecular level. Morphological studies, glass transition phenomena, polymerization kinetics, molecular weight dependence and molecular motion, irradiation effects as well as the size and distribution of free volumes have been examined in this way (see the review given in [2]).

In polystyrene the positron annihilation phenomenon has been studied eagerly from the very beginning of the development of PAS [3-6] up to nowadays $[7,8]$. Positronium (Ps) formation has been confirmed in this polymer by the existence of the long-lived component in positron lifetime spectra $(\tau \approx 2 \mathrm{~ns})$ and its magnetic quenching [9] as well as by the appearance of the narrow component in angular correlation of annihilation radiation (ACAR) curves [6] and the abundance of the three-quantum annihilation events [10]. In the paper [11] a value 
of the glass transition temperature $\left(T_{\mathrm{g}}\right)$ of commercial polystyrene was given as a result of measurements of temperature dependence of the o-Ps pickoff lifetime. The temperature dependence of the lifetime and intensity of $o-P s$ decaying by the pickoff mechanism was interpreted as a free-volume effect. Further insight into the nature of the glass transition in polystyrene has been gained in [12]. The values of $T_{\mathrm{g}}$ determined from positron annihilation lifetime (PAL) measurements increased with the number average molecular weight in the studied range (50100-640000). The variation of the longest lifetime with temperature indicated the onset of the coordinated segmented motion about the polymer axis about $13 \mathrm{deg}$ below the temperature $T_{\mathrm{g}}$ measured on a differential scanning calorimeter. It was established [13] that the pickoff decay time of $a$-Ps in polystyrene remained constant at $\tau \approx 1.9 \mathrm{~ns}$ for the number average molecular weights $\bar{M}_{n}$, within the range $10^{3} \div 2 \times 10^{6}$. For lower molecular weights the longest lifetime increased with decreasing $\bar{M}_{n}$, reflecting thereby the dependence of the free volume on $\bar{M}_{n}$. It was concluded that the correlation frequencies for molecular motion in polystyrene below $T_{\mathrm{g}}$ are too low to affect the long lifetime.

As far as the positronium formation in polystyrene is concerned, one can find a remark in [14] that it takes place mainly in the benzene rings occurring in polystyrene. The relaxation parameter (i.e. the electron density at the position of the positron forming Ps in polystyrene related to that of free Ps) was estimated in [9] as equal to $0.80 \pm 0.06$ indicating the relaxation of the Ps.

\section{Experimental}

\subsection{Samples. Polystyrene and chloropolystyrenes}

Two series of samples were studied. The first one included polystyrene (PSPthe raw material and PSB) and one chloropolystyrene (PO7), while the second the commercial polystyrene (AOP-the raw material) and five chloropolystyrene samples (AOW, AO3, AO4, AO6, AO48). Further characteristics of samples as chlorine content and its distribution are given in Table I. All the studied samples were amorphous as it was proved by X-ray diffraction studies. Powders of each sample, except PSB which was in bulk form, were formed into discs, of diameter equal to $20 \mathrm{~mm}$ and of thickness equal to $3 \mathrm{~mm}$, under the pressure of 3 tons.

\subsection{Polymer preparation and characterization}

The polystyrene samples (PSP and PSB) were obtained by the radical polymerization of styrene in benzene solutions, the procedure described in [16]. The chlorination of polystyrene was carried out with sulphuryl chloride in two ways: (a) radical initiation (samples $\mathrm{PO}, \mathrm{AOW}, \mathrm{AO}, \mathrm{AO} 4, \mathrm{AO}$ ) [17] and (b) ionic catalysis (sample AO48) [18]. The chlorine content was determined using the Schöniger method [19].

The positions of the chlorine atoms in the chlorinated polystyrenes depend on the mechanism of chlorination. In the chloropolystyrenes obtained in radical-initiated reactions, the chlorine atoms were substituted on the aliphatic 
part of the polymer (samples $\mathrm{AOW}, \mathrm{AO}, \mathrm{AO} 4, \mathrm{AO} 6$ ) and as in case of the sample PO7 added to the cyclic aliphatic ring and substituted on the chain. The general formula of samples AOW-AO6 is

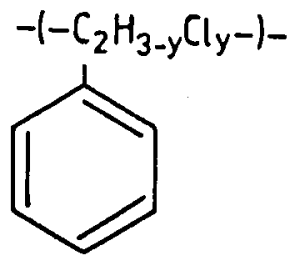

with values of $y$ given in Table I, while the formula of the sample PO7 is

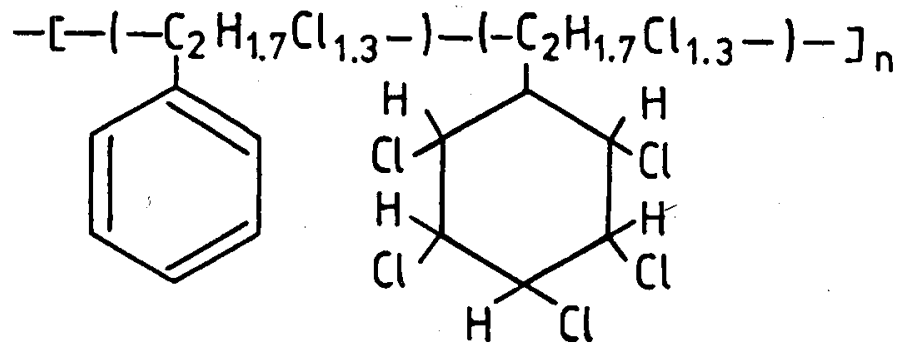

$95 \%$

$5 \%$

TABLE I

Chlorine content in studied samples.

\begin{tabular}{l|c|c|c|c|c}
\hline \hline \multirow{2}{*}{ Sample } & \multicolumn{4}{|c}{ Chlorine content } \\
\cline { 4 - 6 } \multicolumn{2}{c}{} & \multirow{3}{*}{ wt \%] } & \multicolumn{3}{|c}{ [Cl atom/unit] } \\
\cline { 4 - 6 } \multicolumn{1}{c}{} & & $\begin{array}{c}\text { Aliphatic } \\
\text { chain }\end{array}$ & \multicolumn{3}{|c}{ Ring } \\
\cline { 4 - 6 } 1st series & PSP & - & - & - & - \\
& PSB & - & - & - & - \\
& PO7 & 36.1 & 1.3 & - & 0.3 \\
\hline \multirow{6}{*}{ 2nd series } & & $y$ values & & \\
& AOP & - & - & - & - \\
& AOW & 1.1 & 0.03 & - & - \\
& AO3 & 3.6 & 0.1 & - & - \\
& AO4 & 15.3 & 0.5 & - & - \\
& AO6 & 30.8 & 1.3 & - & - \\
& AO48 & 66.0 & 0.6 & 4.9 & -
\end{tabular}


In the chloropolystyrene $\mathrm{AO} 48$, obtained by the ionic chlorination of polystyrene, the chlorine atoms were substituted on the aromatic rings of the polystyrene and on the aliphatic chain. The formula of the sample AO48 is

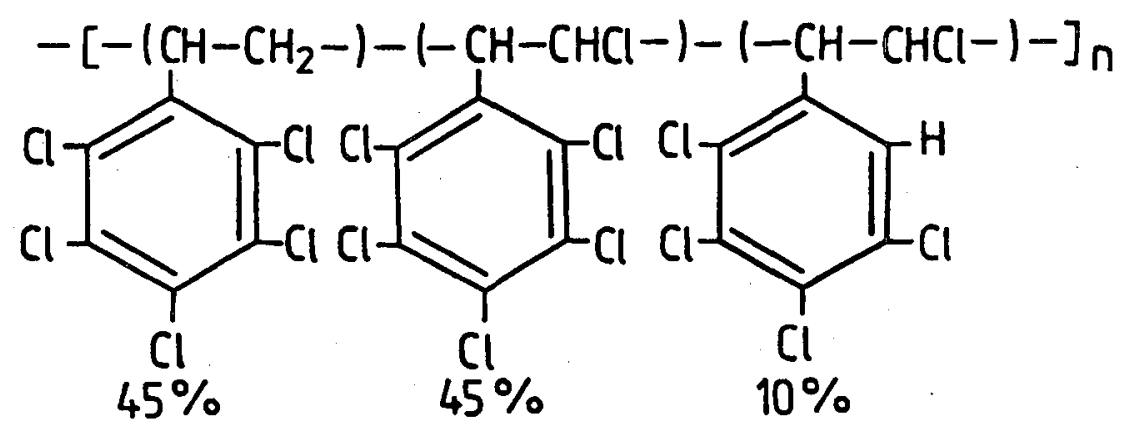

All structures of the chloropolystyrenes were established with the use of the ${ }^{1} \mathrm{H}-\mathrm{NMR}$, IR spectroscopy techniques and elementary analysis [17].

\subsection{Measurements}

ACAR and PAL techniques were used in our studies of polystyrene and chloropolystyrene samples. The two kinds of measurements were performed in air at room temperature.

\subsubsection{ACAR measurements}

ACAR measurements were performed from -29 to $+29 \mathrm{mrad}$ with the use of the LS spectrometer of angular resolution with FWHM equal to $0.8 \mathrm{mrad}$, described in details in [15]. Data were accumulated with the step of $0.5 \mathrm{mrad}$ for the period of $2 \times 10^{3} \mathrm{~s}$ at each point resulting in about $8 \times 10^{4}$ counts at the peak of each distribution with background intensity reaching $3 \%$ of the peak value. The positrons were produced upon desintegration of ${ }^{22} \mathrm{Na}$ nuclei in the source of $15 \mathrm{mCi}$ activity.

\subsubsection{PAL measurements}

A conventional fast-slow coincidence system with plastic, cylindrical scintillators NE $111(20 \mathrm{~mm} \times 20 \mathrm{~mm})$ was used for measuring the lifetime spectra. The positron source, ${ }^{22} \mathrm{Na}$ of activity $15 \mu \mathrm{Ci}$ was deposited between two identical thin hostaphan foils $\left(0.8 \mu \mathrm{C} \cdot \mathrm{cm}^{-2}\right.$ each) and then sandwiched between the samples under investigation. A typical decay curve contained about $1 \times 10^{6}$ counts and had the peak to background ratio of 2500 .

\section{Results}

The ACAR curves were fitted with a sum of Gaussians (for samples PSP, PSB, AOP, AOW, and AO3) or the sum of one very broad Gaussian and a Ferrell function [20] (samples $\mathrm{PO}, \mathrm{AO} 48, \mathrm{AO}$, and AO6) simultaneously together 
with a backgroumd represented as a sloped straight line, using the computer program FER [21]. The correction for the geometrical resolution of the spectrometer was made. The results of the analysis, i.e. the FWHM and the intensity of each component are given in Table II (see next page).

The PAL spectra corrected for positron annihilation in the source $(3 \%)$ were fitted with a sum of three exponential functions convoluted with the resolution function of the lifetime spectrometer (a sum of two Gaussians), plus a constant background, using the program POSITRONFIT [22]. The results of the analysis, i.e. the lifetimes and the intensities are given in Table III. An example of results of

TABLE III

Results of PAL measurements.

\begin{tabular}{c|c|c|c|c|c|c|c}
\hline \hline \multirow{2}{*}{$\begin{array}{c}\text { Sam- } \\
\text { ple }\end{array}$} & \multicolumn{3}{|c|}{ Lifetimes [ps] } & \multicolumn{3}{|c|}{ Intensities [\%] } & \multirow{2}{*}{$\begin{array}{c}\text { Variance } \\
\text { of the fit }\end{array}$} \\
\cline { 2 - 7 } & $\tau_{1}$ & $\tau_{2}$ & $\tau_{3}$ & $I_{1}$ & $I_{2}$ & $I_{3}$ & 1.08 \\
PSP & $122(5)^{*}$ & $418(10)$ & $2059(13)$ & $27.9(1.1)$ & $36.0(0.9)$ & $36.6(0.3)$ & 1.08 \\
PSB & $134(5)$ & $407(8)$ & $2005(8)$ & $27.1(1.0)$ & $34.9(0.9)$ & $38.1(0.2)$ & 0.92 \\
PO7 & $158(15)$ & $389(4)$ & $2174(120)$ & $14.6(1.8)$ & $83.6(1.7)$ & $1.8(0.1)$ & 0.92 \\
\hline AOP & $128(7)$ & $411(17)$ & $2012(15)$ & $31.8(1.6)$ & $28.6(1.4)$ & $39.6(0.4)$ & 1.08 \\
AOW & $132(6)$ & $382(5)$ & $2005(11)$ & $21.3(1.0)$ & $56.7(1.0)$ & $22.0(0.2)$ & 0.95 \\
AO3 & $148(10)$ & $374(5)$ & $2003(15)$ & $17.4(1.6)$ & $65.9(1.5)$ & $16.7(0.2)$ & 1.03 \\
AO4 & $165(12)$ & $379(5)$ & $2064(24)$ & $18.5(2.1)$ & $72.0(1.9)$ & $9.5(0.2)$ & 1.03 \\
AO6 & $210(12)$ & $403(7)$ & $2359(65)$ & $25.3(3.4)$ & $71.3(3.3)$ & $3.4(0.1)$ & 0.96 \\
AO48 & $213(14)$ & $403(8)$ & $1897(54)$ & $25.7(4.1)$ & $70.5(4.0)$ & $3.8(0.2)$ & 1.04 \\
\hline
\end{tabular}

* Standard deviations of measured quantities are given in round brackets.

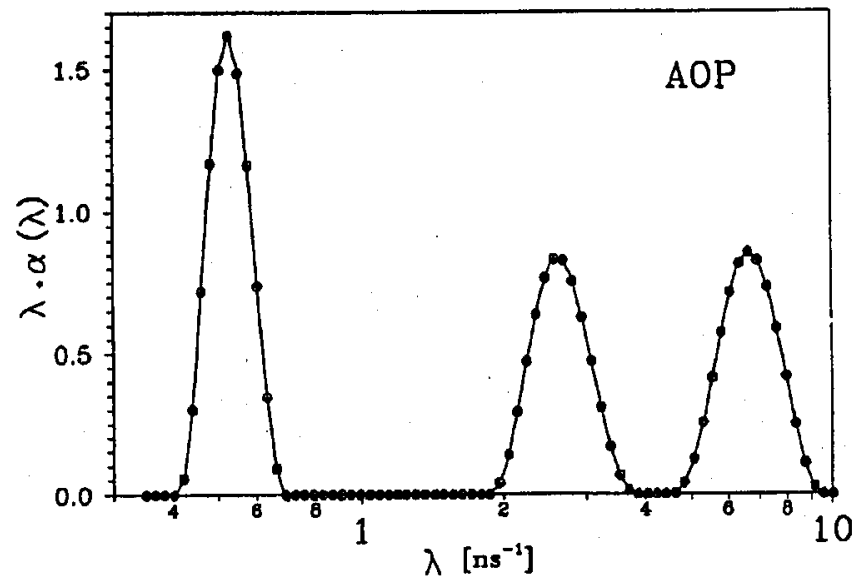

Fig. 1. The annihilation rate distribution for positrons annihilating in AOP. 


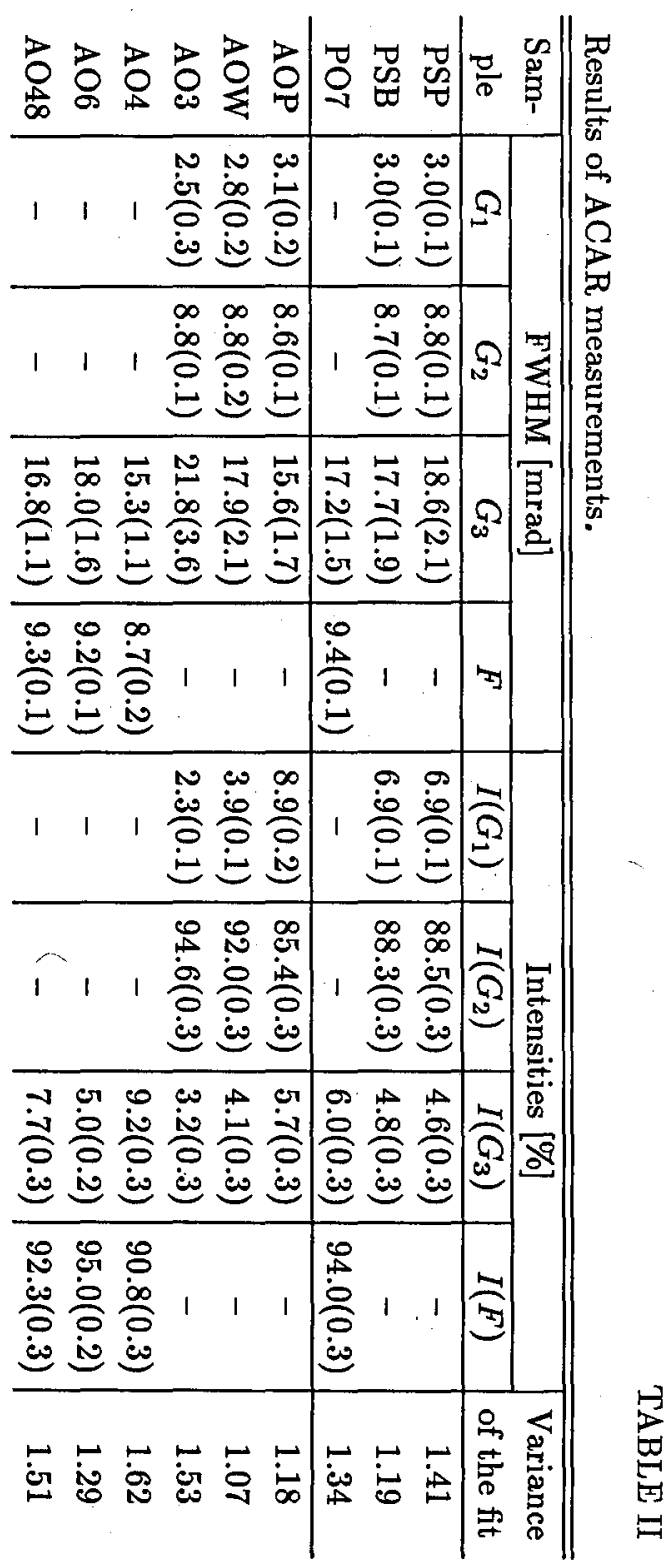


analysis of PAL data with the use of a new method employing numerical Laplace inversion techniques [23] is given for the commercial polystyrene (sample AOP). The program CONTIN was used to obtain the annihilation rate $(\lambda)$ distribution as well as the radius $(R)$ and volume $(V)$ distributions of free-volume cavities in the polystyrene, given in Figs. 1, 2 and 3 . Further details concerning the method are given in Appendix. The values of $\lambda$ and intensities of the maxima in the $\lambda$ distribution.correspond quite well with the lifetime data for this sample.

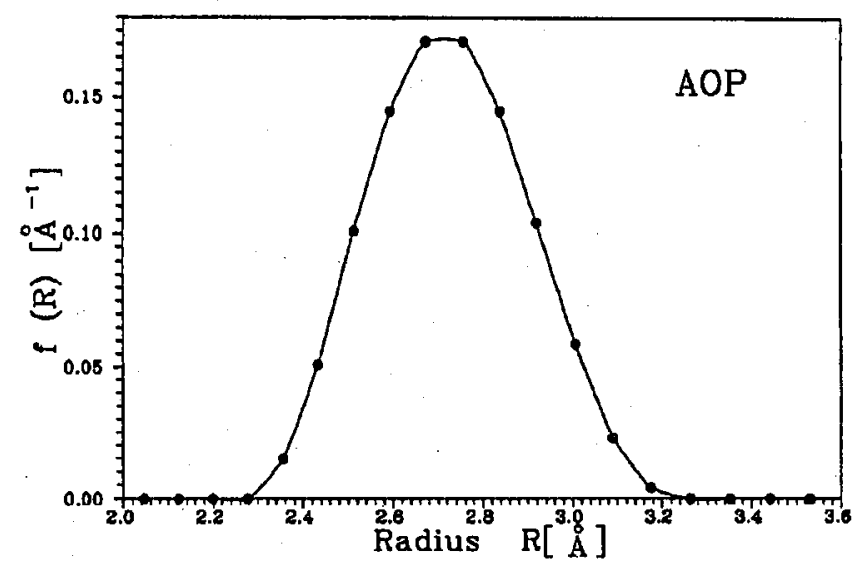

Fig. 2. The radius distribution of free volume cavities in AOP assuming a spherical cavity with radius $R$ determined from the ortho-positronium annihilation rate according to the formula (3) given in Appendix.

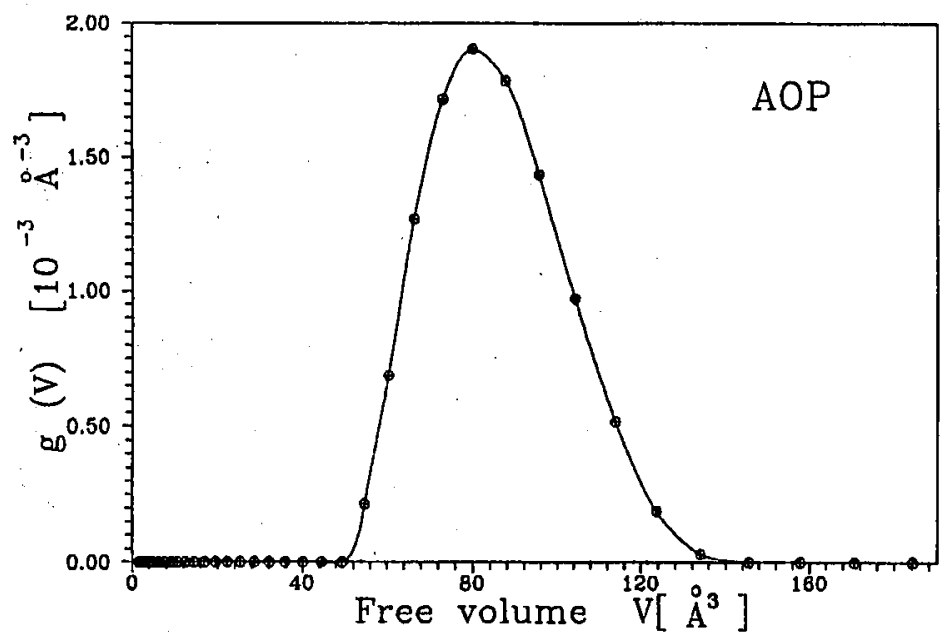

Fig. 3. The volume distribution of free volume cavities in AOP. 


\section{Discussion}

Comparison of the results for PSP and PSB samples indicates that there is no essential difference in positron annihilation characteristics for powder and bulk polystyrene. The lifetimes for raw samples in both series (PSP and AOP) are very close to those known from literature [7] and being the results of the high-precision measurements.

Assuming that in polystyrene the longest-lived component in the lifetime spectra $\left(\tau_{3}, I_{3}\right)$ comes from the ortho-positronium atoms decaying via the pickoff process, one can conclude that the positronium formation in chloropolystyrenes is strongly inhibited. The inhibition reveals even at the low content of $\mathrm{Cl}$ atoms (the large decrease in $I_{3}$ follows the little increase in the chlorine content).

In Fig. 4 the decrease in $I_{3}$ is given vs. $\mathrm{Cl}$ content for samples of both series. When one accepts the values of $\Delta I_{3}$ obtained for the samples of the second series

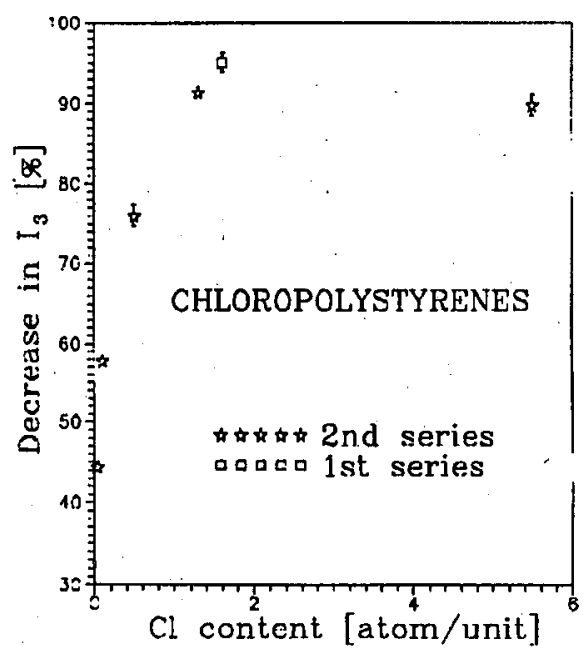

Fig. 4. The decrease in $I_{3}$ vs. Cl content.

as reflecting the inhibition possibility of $\mathrm{Cl}$ atoms in the aliphatic chain, one can count the additional decrease in $I_{3}$ which takes place in the samples PO7 and is caused by the chlorine atoms attached to the cyclic aliphatic ring sand AO48 caused by the $\mathrm{Cl}$ atoms in benzene rings. From rough estimation the values of $\Delta I_{3}$ equal to $12 \%$ and $2.2 \%$ per one $\mathrm{Cl}$ atom situated as mentioned above in the polymer structure in PO7 and AO48 are obtained respectively. It is obvious that the $\mathrm{Cl}$ atoms attached to aliphatic carbons are more active in the inhibition of Ps formation than those substituted on the aromatic rings in AO48 sample.

The general conclusion is that the chlorine inhibits the positronium formation but its "activity" towards positrons depends on its position in the polymer structure, this means on the kind of bond between the halogen and carbon.

The intensities $I_{3}$ vs. chlorine content $(C)$ for the samples of the second series are well fitted with the equation: $I_{3}=I_{3}^{0}\left[1+(\alpha C)^{\beta}\right]^{-1}$, where $I_{3}^{0}$ denotes the $\alpha$-Ps 
yield in the polystyrene sample (AOP), while $\alpha$ and $\beta$ are the fitting parameters equal respectively to $0.55[\mathrm{wt} \%]^{-1}$ and 0.63 (Fig. 5). The above equation has

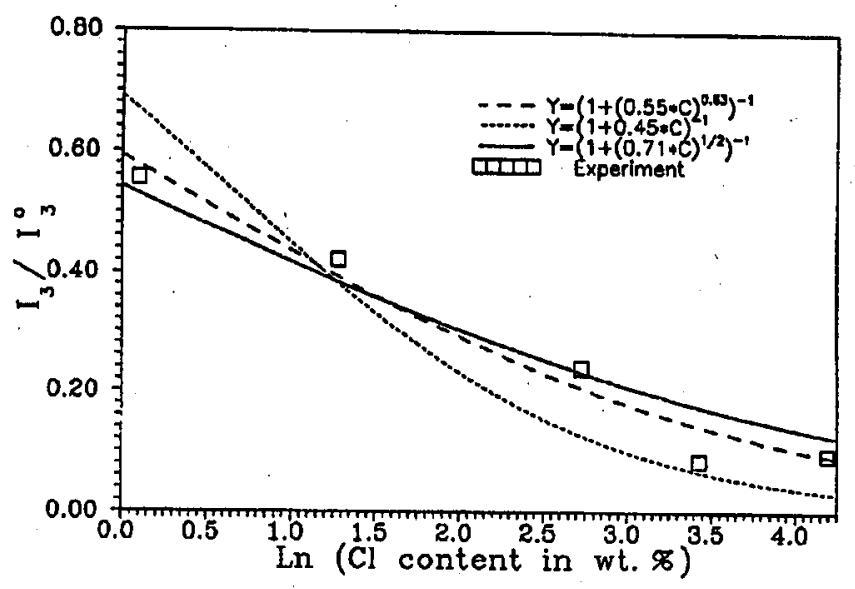

Fig. 5. $I_{3} / I_{3}^{0}$ vs. $\mathrm{Cl}$ content. The experimental data were fitted with the three general formulae: $I_{3}=I_{3}^{0}\left[1+(A C)^{B}\right]^{-1}, I_{3}=I_{3}^{0}[1+A C]^{-1}$ and $I_{3}=I_{3}^{0}\left[1+(A C)^{1 / 2}\right]^{-1}$ using the method of least squares. The fitting parameters $A$ and $B$ are given above.

been proposed to represent the o-Ps yield versus the concentration of electron scavengers in solutions [24]. It is based on the assumption that the relative yield of $\mathrm{Ps}$ is proportional to the relative fraction of geminate electrons which remained unscavenged by the introduced electron scavenger.

According to the spur model the Ps yield in polymers should be large due to the small size of the spur in solids at all, and the high values of dielectric constants in polymers. Relative high contributions of the spur mechanism in the Ps formation were estimated in [25] for some polymers.

Interpreting the observed inhibition of positronium in the frame of the spur model of Ps formation one may take into account the possibility of the $\left[\mathrm{Cl}^{-}, \mathrm{e}^{+}\right]$ bound state formation. The existence of such state has been proposed many times in case of samples containing $\mathrm{Cl}$ and studied with the use of PAS, e.g. in aqueous solutions of $\mathrm{Cl}^{-}$ions [26], in chlorine substituted hydracarbons in non-polar liquids [27], in polyvinyl chloride on thermal degradation [28], in fluorinated elastomers with $\mathrm{Cl}$ in side groups [29] and for alkali chlorides [30]. In Ref. [30] the stability of the $\left[\mathrm{Cl}^{-}, \mathrm{e}^{+}\right]$bound state was established and its main characteristics were given, e.g. the full width at half maximum of the ACAR curve $(11.2 \mathrm{mrad})$ as well as the positron lifetime (328 ps). Thus, if the complex $\left[\mathrm{Cl}^{-}, \mathrm{e}^{+}\right]$is formed, it does not contribute to the long lifetime in the PAL spectra and to the narrow component in the ACAR curves. The changes in positron annihilation characteristics abserved by us versus chlorine content do not contradict the supposition that the formation of the $\left[\mathrm{Cl}^{-}, \mathrm{e}^{+}\right]$bound state is the reason of the Ps inhibition in the chloropolystyrenes. 


\section{Appendix}

In Refs. [23, 31] a method for the analysis of PAL data was given in which the count function, $C(t)$ was represented as the Laplace transform of the annihilation rate probability density function (pdf), $\alpha(\lambda)$ :

$$
C(t)=\lambda \alpha(\lambda) \exp (-\lambda t) \mathrm{d} \lambda=L\{\lambda \alpha(\lambda)\}:
$$

$\alpha(\lambda) \mathrm{d} \lambda$ gives the fraction of positrons annihilating with rates between $\lambda$ and $\lambda+\mathrm{d} \lambda$.

A modified version of Provencher's program, CONTIN [1], enables to recover $\alpha(\lambda)$ by numerical Laplace inversion. Further modifications to the program to correct the source terms in the sample and reference decay curves and for shifts in the position of the zero-time channel of the sample and reference data were introduced by Gregory and described in [32].

Transformation of the annihilation rate $\mathrm{pdf}, \alpha(\lambda)$, into the free-volume radius pdf, $f(R)$, is based on the following assumptions:

1. $o$-Ps atom is localised in a spherical free-volume region of radius $R$.

2. o-Ps annihilates via pickoff in a homogeneous electron layer of thickness $\Delta R$ inside the wall surrounding the region of the free volume. The o-Ps annihilation rate inside the electron layer is assumed to be equal to $2 \mathrm{~ns}^{-1}$.

3. The sum of $R+\Delta R$ is equal to $R_{0}$ which denotes the size of a spherical well of the infinite and square potential.

As a result of the approach given above, originally presented by Tao [33] and Eldrup et al. [34], one obtains the expression (2) for $\lambda$ :

$$
\lambda=2\left\{1-R /(R+\Delta R)+(2 \pi)^{-1} \sin [2 \pi R /(R+\Delta R)]\right\},
$$

where $\Delta R=1.656 \AA$ is an empirical parameter obtained by fitting the measured o-Ps annihilation rates in materials of well-defined empty spaces (e.g. pores in zeolites) [35].

Thus, the free-volume radius pdf, $f(R)$, is

$$
f(R)=-2 \Delta R\{\cos [2 \pi R /(R+\Delta R)]-1\} \alpha(\lambda) /(R+\Delta R)^{2}
$$

and the corresponding free volume pdf, $g(V)$, is

$$
g(V)=f(R) / 4 \pi R^{2} .
$$

\section{Acknowledgment}

We are grateful to Dr. R.B. Gregory for making a copy of CONTIN available.

This work was supported by grant from the Ministery of National Education 105/E-319/92.

\section{References}

[1] S.W. Provencher, Compt. Phys. Commun. 27, 229 (1982).

[2] H.J. Ache, in: Positron and Positronium Chemistry, Eds. D.M. Schrader, Y.C. Jean, Elsevier, Amsterdam 1989, p. 318, or: Y.C. Jean, Microchem. J. 42, 72 (1990). 
[3] S. De Benedetti, H.J. Richings, Phys. Rev. 85, 377 (1952).

[4] R.E. Bell, R.L. Graham, Phys. Rev. 87, 236 (1952).

[5] R.E. Bell, R.L. Graham, Phys. Rev. B 90, 644 (1953).

[6] L.A. Page, M. Heinberg, Phys. Rev. 102, 1545 (1956).

[7] J.E. Kluin, F. Faupel, Mater. Sci. Forum 105-110, 1613 (1992).

[8] M. Welander, F.H.J. Mauer, in Ref. [7], p. 1811.

[9] M. Bertolaccini, A. Bisi, G. Gambarini, L. Zappa, J. Phys. C, Solid State Phys. 7; 3827 (1974).

[10] M. Bertolaccini, C. Bussolati, L. Zappa, Phys. Rev. A 139, 696 (1965).

[11] B.C. Groseclose, G.D. Loper, Phys. Rev. A 137, 939 (1965).

[12] J.R. Stevens, A.C. Mao, J. Appl. Phys. 41, 4273 (1970).

[13] D.H.D. West, V.J. McBrierty, C.F.G. Delaney, Appl. Phys. 7, 171 (1975).

[14] B.V. Thosar, V.G. Kulkarni, R.G. Lagu, G. Chandra, Phys. Lett. 21, 647 (1966).

[15] B. Rozenfeld, A. Baranowski, K. Jerie, Nukleonika 19, 817 (1974).

[16] I. Losev, O. Fedotova, Praktikum po khimii vysokomolekularnȳkh soedinenii, Goskhimizdat, Moskva 1962 (in Russian).

[17] G. Mirkiewicz, Ph.D. thesis, to be published.

[18] R.T. Sikorski, G. Mirkiewicz, Eur. Polym. J. 24, 157 (1988).

[19] J. Pielichowski, A. Puszyński, Preparatyka zwiqzków wielkoczasteczkowych, Politechnika Krakowska, Kraków 1978, p. 142 (in Polish).

[20] A. Baranowski, E. Dębowska, Appl. Phys. A 51, 23 (1990).

[21] A. Baranowski, Program FER, in a software of the Computer Library of the Department of Applied Nuclear Physics at Wroclaw University.

[22] P. Kirkegaard, N.J. Pedersen, M. Eldrup, PATFIT-88: A Data Processing System for Positron Annihilation Spectra on. Mainfame and Personal Computers, Tech. Rep. RIS $\varnothing-M-2740$, Ris $\emptyset$ National Laboratory, Roskilde 1989.

[23] R.B. Gregory, Y. Zhu, Nucl. Instrum. Methods Phys. Res. A 290, 172 (1990).

[24] References [32] and [33] in G. Wikander, O.E. Mogensen, N.J. Pedersen, Chem. Phys. 77, 159 (1983).

[25] A. Bisi, G. Gambarini, L. Zappa, Nuovo Cimento D 2, 1465 (1983).

[26] O.E. Mogensen, Chem. Phys. 37, 139 (1979).

[27] G. Wikander, O.E. Mogensem, J. Pedersen, Chem. Phys. 87, 149 (1984).

[28] M.N.G.A. Khan, J. Phys. D 3, 663 (1970).

[29] P.U. Arifov, S.N. Vasserman, S.A. Tishin, in: Positron Annihilation, Eds. P.C. Jain, R.M. Singru, K.P. Gopinathan, World Scientific, Singapore 1985, p. 787.

[30] S.B. Nuramagambetov, in Ref. [29], p. 770.

[31] R.B. Gregory, A. Procyk, in: Positron Annihilation Studies of Fluids, Ed. S.C. Sharma, World Scientific, Singapore 1988, p. 254.

[32] R.B. Gregory, Nucl. Instrum. Methods Phys. Res. A 302, 496 (1991).

[33] S.J. Tao, J. Chem. Phys. 56, 5499 (1972).

[34] M. Eldrup, D. Li i.ltbody, J.N. Sherwood, Chem. Phys. 63, 51 (1981).

[35] H. Nakaniski, S.J. Wang, Y.C. Jean, in Ref. [31], p. 292. 